\title{
Transient Loads on Buildings in Microburst and Tornado Winds
}

\author{
Anindya Sengupta, Fred L. Haan, Partha P. Sarkar and Vasanth Balaramudu \\ Department of Aerospace Engineering, 2271 Howe Hall, Iowa State University, Ames, IA USA
}

\begin{abstract}
This paper presents both quasi-steady and transient wind load effects on a cubic building in a microburst and a tornado. Large Eddy Simulation (LES) was used to simulate the loading effects of a translating microburst with a 0.203 m-diameter jet at four different translating velocities on a $25.4 \mathrm{~mm}$ cube. The results of the numerical simulation were compared with data from a model laboratory microburst simulator. The transient loading effects on a 228.6 $\mathrm{mm}$ cubic building from a translating tornado were also simulated for two different vortex core diameters and two translating speeds and the peak loads were studied.
\end{abstract}

KEYWORDS: microburst, tornado, LES, peak loads, transient effects, cubic building.

\section{INTRODUCTION}

Tornadoes are vortices with significant rotational velocity along with vertical and radial velocity components in the core region. Physical simulations of tornadoes have been carried out by Ward [1] and Church et al. [2] and numerical simulation has been done by Lewellen [3]. Microbursts are characterized by a strong localized down-flow and an outburst of strong winds near the surface. Selvam and Holmes [4] and Wood et al. [5] have conducted numerical and experimental simulations of thunderstorm downdrafts using static impinging jet models. Both physical and numerical simulations of tornado and microburst to study their effects on scaled models of buildings have been conducted by Bienkiewicz and Dudhia [6], Letchford and Chay [7], Sengupta and Sarkar [8], and Sarkar et al. [9].

\section{NUMERICAL SIMULATION}

Large Eddy Simulation (LES) was used to simulate the effects of a translating microburst with a $0.203 \mathrm{~m}$-diameter impinging jet and different jet translating velocities on a $25.4 \mathrm{~mm}$ cube. The dynamic Smagorinsky-Lilly LES model was used for studying the steady and moving jet impingement flow characteristics with emphasis on the effects of this kind of flow on a cubic building. A central difference scheme was used for the convective terms and for pressure correction the PISO algorithm was used. The jet nozzle was moved at four different speeds, $0.225 \mathrm{~m} / \mathrm{s}(\mathrm{S} 1), 0.5 \mathrm{~m} / \mathrm{s}(\mathrm{S} 2), 1 \mathrm{~m} / \mathrm{s}(\mathrm{S} 3)$ and $2 \mathrm{~m} / \mathrm{s}(\mathrm{S} 4)$ using the sliding mesh simulation method. The simulations were carried out using Fluent [10], with varying domain sizes and time steps.

\section{EXPERIMENTAL SETUP}

\subsection{Microburst Simulator}

A nozzle diameter (D) of $203 \mathrm{~mm}$ was used to produce the impinging jet. The nozzle along with the fan was moved using a garage door opener with a speed of $0.225 \mathrm{~m} / \mathrm{s}(\mathrm{S} 1)$, to simulate a translating microburst. The distance $(\mathrm{H})$ of the ground plane or impinging platform from the nozzle could be varied from a minimum of $203 \mathrm{~mm}$ to a maximum of $826 \mathrm{~mm}$ using a mechani- 
cal jack. For the current work, $\mathrm{H}=403 \mathrm{~mm}, \mathrm{H} / \mathrm{D}=1.98$ and a jet velocity, $\mathrm{V}_{\mathrm{j}} \approx 10 \mathrm{~m} / \mathrm{s}$ was used. Surface pressures on the $25.4-\mathrm{mm}$ cube, oriented normally to the direction of translation, were measured using Scanivalve pressure transducers.

\subsection{Tornado Simulator}

Measurements were made for two tornado vortex cases, with core radii of $0.31 \mathrm{~m}(\mathrm{R} 1)$ and 0.56 $\mathrm{m}(\mathrm{R} 2)$, each of which had a maximum tangential velocity of $11.0 \mathrm{~m} / \mathrm{s}$. Swirl ratios for these two cases were estimated to be 0.39 and 1.42 . Here $\mathrm{D}$ is defined as twice the core radius.

Aerodynamic forces were measured on a 1:100 scale model of a 228.6-mm cubic building which was constructed with plywood. The force balance that was used is capable of measuring all three forces and all three moment components. Two building orientations, normal and 45 degrees with respect to the tornado translation axis, were tested. For both orientations, three different cases of tornado translation were considered. The tornado was translated at $0.30 \mathrm{~m} / \mathrm{s}$ (LS) and $0.61 \mathrm{~m} / \mathrm{s}$ (HS). For the quasi-steady (QS) case, the tornado was held at specific distances from the model.

\section{RESULTS AND DISCUSSION}

Figures $1 \mathrm{a}$ and $1 \mathrm{~b}$ show the comparison of numerical and experimental transient drag and lift coefficients on the 25.4-mm cubic building due to a translating microburst for Speed S1.
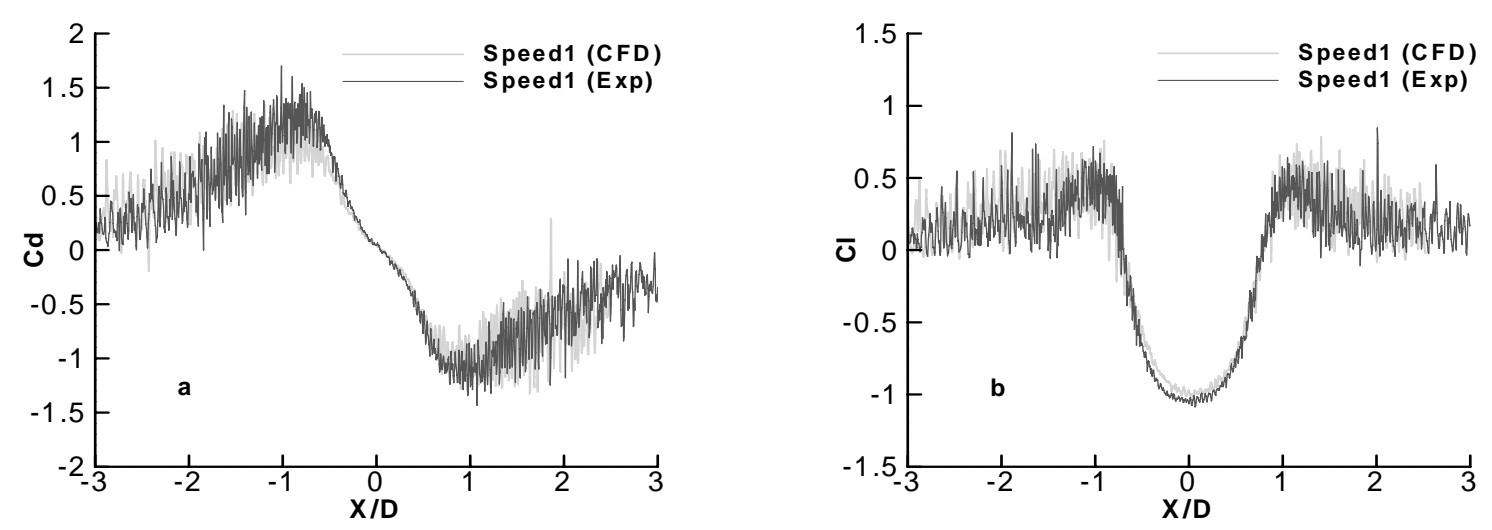

Figure1. (a) Drag coefficient for $25.4 \mathrm{~mm}$ cube (b) Lift coefficient for $25.4 \mathrm{~mm}$ cube
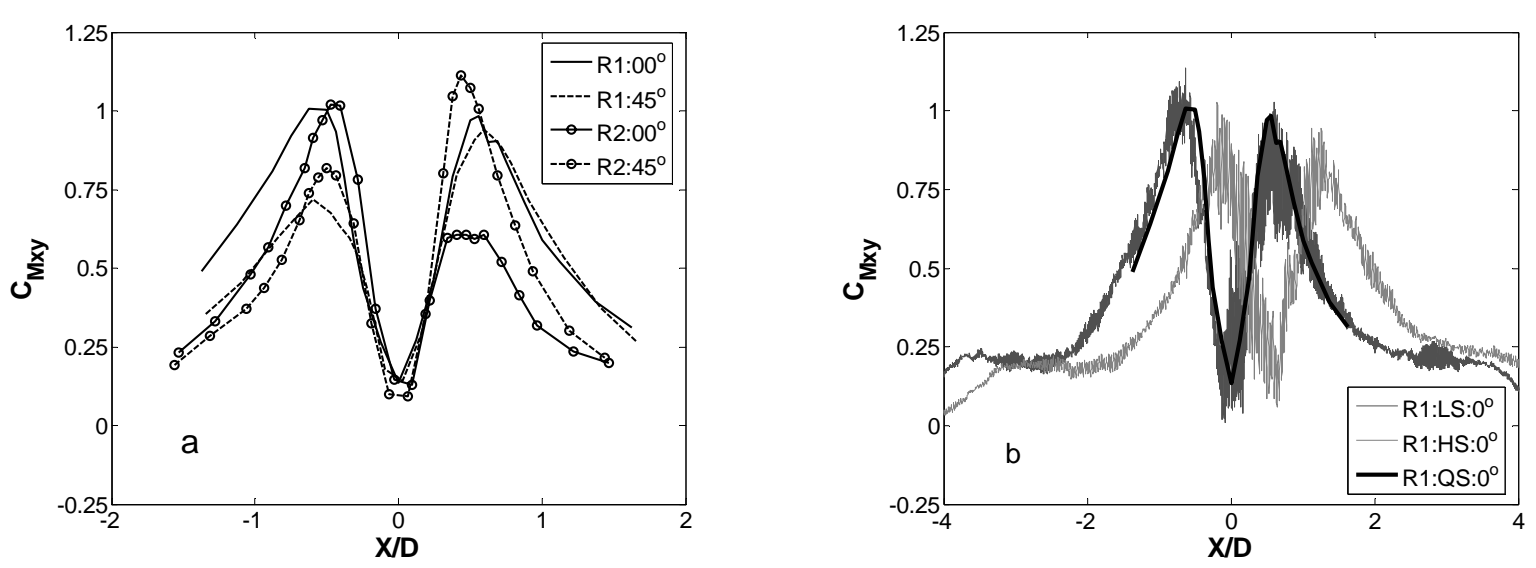

Figure2. (a) Moment coefficient comparison for various building orientations and vortex radii

(b) Moment coefficient comparison for QS, LS and HS. 
Table1. Summary of peak force and moment coefficients for all tornado and microburst cases

\begin{tabular}{|c|c|c|c|c|c|c|c|c|c|c|}
\hline \multicolumn{7}{|c|}{ Tornado } & \multicolumn{4}{|c|}{ Microburst } \\
\hline \multirow{2}{*}{ 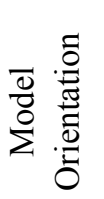 } & \multirow{2}{*}{ 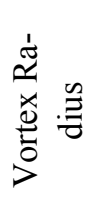 } & \multirow{2}{*}{ 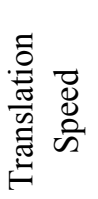 } & \multicolumn{2}{|c|}{ Force } & \multicolumn{2}{|c|}{ Moment } & \multirow{2}{*}{\multicolumn{2}{|c|}{ 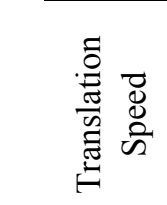 }} & \multicolumn{2}{|c|}{ Force } \\
\hline & & & $\mathrm{C}_{\mathrm{Fxy}}$ & $\mathrm{C}_{\mathrm{Fz}}$ & $\mathrm{C}_{\mathrm{Mxy}}$ & $\mathrm{C}_{\mathrm{Mz}}$ & & & $\mathrm{C}_{\mathrm{D}}$ & $\mathrm{C}_{\mathrm{L}}$ \\
\hline \multirow{6}{*}{$0^{\circ}$} & \multirow{3}{*}{ R1 } & QS & 1.80 & 1.26 & 1.01 & 0.20 & \multirow{4}{*}{ 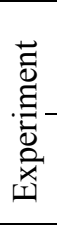 } & \multirow{2}{*}{ QS } & \multirow{2}{*}{1.70} & \multirow{2}{*}{-1.10} \\
\hline & & LS & 1.97 & 1.44 & 1.14 & 0.30 & & & & \\
\hline & & HS & 1.79 & 1.18 & 1.03 & 0.22 & & S1 & 170 & 107 \\
\hline & \multirow{3}{*}{ R2 } & QS & 1.87 & 1.11 & 1.02 & 0.11 & & & & \\
\hline & & LS & 1.97 & 1.24 & 1.10 & 0.24 & \multirow{5}{*}{ 穵 } & QS & 1.40 & -1.00 \\
\hline & & HS & 1.82 & 1.22 & 1.04 & 0.16 & & S1 & 1.40 & -1.03 \\
\hline \multirow{6}{*}{$45^{\circ}$} & \multirow{3}{*}{ R1 } & QS & 1.53 & 0.97 & 0.94 & 0.14 & & S2 & 1.50 & -1.03 \\
\hline & & $\mathrm{LS}$ & 1.25 & 1.13 & 0.79 & 0.31 & & S3 & 1.70 & -1.03 \\
\hline & & $\mathrm{HS}$ & 1.36 & 1.19 & 0.86 & 0.29 & & S4 & 2.00 & \pm 1.10 \\
\hline & \multirow{3}{*}{$\mathrm{R} 2$} & QS & 1.88 & 1.08 & 1.11 & 0.13 & \multirow{5}{*}{\multicolumn{4}{|c|}{$\begin{array}{l}\mathrm{x}-\mathrm{y}-\mathrm{z} \text { : right-hand-coordinate } \\
\text { system; } \mathrm{x} \text { : direction of } \\
\text { translation; } \mathrm{y} \text { : normal to the } \\
\text { direction of translation; } \mathrm{z} \text { : } \\
\text { positive upwards; } \mathrm{C}_{\mathrm{D}}=\text { positive } \\
\text { along } \mathrm{x} ; \mathrm{C}_{\mathrm{L}}=\text { positive along } \mathrm{z}\end{array}$}} \\
\hline & & $\mathrm{LS}$ & 1.57 & 1.09 & 0.95 & 0.34 & & & & \\
\hline & & HS & 1.40 & 0.98 & 0.83 & 0.30 & & & & \\
\hline \multirow{2}{*}{$\begin{array}{l}\text { Max } \\
\text { Loads }\end{array}$} & \multicolumn{2}{|c|}{ Cube } & 1.97 & 1.44 & 1.14 & 0.34 & & & & \\
\hline & \multicolumn{2}{|c|}{ Tall-Building } & 2.17 & 1.78 & 1.15 & 0.53 & & & & \\
\hline
\end{tabular}

The force coefficient values, as in Table 1, corresponding to the CFD cases (QS and S1) are smaller than the experimental cases because the values for the latter were calculated based on the centerline pressures only. The mean pressure coefficient values along the centerline of the model matched quite well between CFD and experimenatal cases. The drag coefficient $C_{D}$ was observed to significantly increase while the lift coefficient $C_{L}$ does not show the same pattern with increasing translation speed, as observed in the CFD results.

All force and moment coefficients for the tornado cases were normalized using the maximum tangential velocity of a stationary tornado and the projected area (A) of the model on a plane normal to the translating direction ( $\mathrm{x}$ ) of the tornado for $\mathrm{C}_{\mathrm{Fxy}}, \mathrm{C}_{\mathrm{Mxy}}$, as in Equation 1, or $\mathrm{C}_{\mathrm{Mz}}$ and the top face of the model for $\mathrm{C}_{\mathrm{Fz}}$ with $\mathrm{H}$ defined as height of the model.

$C_{F_{x y}}=\frac{\sqrt{F_{x}^{2}+F_{y}^{2}}}{1 / 2 \rho V_{\theta_{\max }^{2}}^{2} A}$

$$
C_{M_{x y}}=\frac{\sqrt{M_{x}^{2}+M_{y}^{2}}}{1 / 2 \rho V_{\theta_{\max }^{2}}^{2} A H}
$$

$\mathrm{C}_{\mathrm{Fxy}}$ and $\mathrm{C}_{\mathrm{Mxy}}$ are coefficients of the vector sums (in the $x$ and $y$ directions) of forces and moments, respectively. This combination is important for tornado winds because of the three dimensional nature of the flow field. The coefficients for the microburst cases were for single force components only that were normalized similarly except with the jet exit velocity.

Figure 2a shows moment coefficient records for a stationary tornado (QS) at various distances from the center of the building for two different building orientations and two tornado vortex 
core radii. Each quasi-steady data point corresponds to a 30 second average. Figure $2 b$ shows the effect of translation speed of the tornado on the moment coefficients for zero-degree building orientation case. Each laboratory case of microburst and tornado translation corresponds to an average of five identical runs.

The results of all the tests are summarized in Table 1. The peak force and moment coefficients are listed for each case. For each force or moment quantity and each extreme wind scenario, Table 1 shows the maximum peak values in boldface. It is noteworthy that the tornadoinduced loads are maximum not for the fastest translation speed but rather for the half-full speed in most cases. Also, the tornado scenarios produced larger force and moment coefficients than the microburst cases. In addition, tornado produced biaxial bending and a twisting moment and a microburst produced a downward force on the roof which is usually not considered in current design practice. The absolute maximum peak force and moment coefficients for all cases of tornado were compared between the cubic building and a tall building (1:500 scale model, $432 \mathrm{~mm}$ height, 108-mm square plan, as in Sarkar et al. 2006) in Table 1. It was found that $\mathrm{C}_{\mathrm{Fxy}}$ and $\mathrm{C}_{\mathrm{Mxy}}$ peak coefficients were very consistent between the two buildings while $\mathrm{C}_{\mathrm{Fz}}$ and $\mathrm{C}_{\mathrm{Mz}}$ were higher for the tall building.

\section{CONCLUSIONS}

The goal of the work presented here was to subject a cubic building model to simulated tornado and microburst winds to quantify the resulting aerodynamic loading on the building. The peak force and moment coefficients for the tornado cases were found to be higher than those of the microburst and the slowly moving and smaller-sized tornado produced generally higher peak loads. Peak drag coefficient was observed to increase with higher microburst translation speed.

\section{REFERENCES}

1 N.B. Ward, The exploration of certain features of tornado dynamics using a laboratory model, J. of the Atmospheric Sciences, 29 (1972) 1194-1204.

2 C.R. Church, J.T. Snow, G.L. Baker and E. M. Agee, Characteristics of tornado-like vortices as a function of swirl ratio: A laboratory investigation, J. Atmos. Sci., 36 (1979) 1755-1776.

3 W. S. Lewellen, Tornado vortex theory, in Church et al., (Eds.), The Tornado: Its Structure, Dynamics, Prediction, and Hazards, Geophysical Monograph 79, American Geophysical Union, 1993, 19-39.

4 R.P. Selvam and J.D. Holmes, Numerical simulation of thunderstorm downdrafts, J. Wind Eng.Ind. Aerodyn. 41-44 (1992) 2817-2825.

5 G.S. Wood, C.S. Kwok, N.A. Motteram and D.F. Fletcher, Physical and Numerical Modeling of Thunderstorm Downbursts, J. Wind Eng.Ind. Aerodyn. 89 (2001) 535-552.

6 B. Bienkiewicz and P. Dudhia, Physical Modeling of Tornado-Like Flow and Tornado Effects on Building Loading, Proc. 7th U.S. National Conf. on Wind Engineering, 1993, 95-106.

7 C.W. Letchford and M.T. Chay, Pressure distributions on a cube in a simulated thunderstorm downburst. Part B: moving downburst observations, J. Wind Eng.Ind. Aerodyn. 90 (2002) 733-753.

8 A. Sengupta and P.P. Sarkar, Physical and Numerical Simulation of Microburst-Like Wind: A Study of Flow Characteristics and Surface Pressures on a Cube, Proc. 3rd Indian National Conf. on Wind Engineering, Kolkata, India, 2006.

9 P.P. Sarkar, F.L. Haan, V. Balaramudu and A. Sengupta, Laboratory Simulation of Tornado and Microburst to assess Wind loads on Buildings, Proc. ASCE Structures Congress, St. Louis, 2006.

10 Fluent, FLUENT User’s Guide, Release 6.2, Fluent, Inc., Lebanon, New Hampshire, 2005. 